University of Wollongong

Research Online

Faculty of Informatics - Papers (Archive)

Faculty of Engineering and Information

Sciences

October 2007

\title{
MAC Design and Analysis for Wireless Sensor Networks with Co-operative Localisation
}

M. Rengasamy

University of Wollongong

E. Dutkiewicz

University of Wollongong, eryk@uow.edu.au

M. Hedley

CSIRO ICT Centre, Sydney

Follow this and additional works at: https://ro.uow.edu.au/infopapers

Part of the Physical Sciences and Mathematics Commons

\section{Recommended Citation}

Rengasamy, M.; Dutkiewicz, E.; and Hedley, M.: MAC Design and Analysis for Wireless Sensor Networks with Co-operative Localisation 2007.

https://ro.uow.edu.au/infopapers/648

Research Online is the open access institutional repository for the University of Wollongong. For further information contact the UOW Library: research-pubs@uow.edu.au 


\title{
MAC Design and Analysis for Wireless Sensor Networks with Co-operative Localisation
}

\author{
Abstract \\ Abstract-Co-operative localisation in wireless sensor networks (WSNs) is a method in which wireless \\ sensor nodes interact with each other as peers to determine their physical position. In such networks it is \\ crucial for communication between nodes to be contention free to meet the strict timing requirements. \\ Medium Access Control (MAC) protocols provide the strongest influence over contention control in \\ WSNs. The focus of this work is the design and analysis of a WSN MAC protocol suitable for supporting \\ co-operative localisation. This paper presents a design overview of the MAC protocol and details its \\ control structure. Preliminary simulation results are also presented to evaluate network formation \\ aspects of the protocol.

\section{Disciplines} \\ Physical Sciences and Mathematics

\section{Publication Details} \\ This conference paper was originally published as Rengasamy, M, Dutkiewicz, E, Hedley, M, MAC Design \\ and Analysis for Wireless Sensor Networks with Co-operative Localisation, International Symposium on \\ Communications and Information Technologies ISCIT 2007, 17-19 Oct, 942-947.
}




\title{
MAC Design and Analysis for Wireless Sensor Networks with Co-operative Localisation
}

\author{
(Rajan) Mohan Rengasamy ${ }^{*}$, Eryk Dutkiewicz ${ }^{*}$ and Mark Hedley ${ }^{\dagger}$ \\ ${ }^{*}$ School of Electrical, Computer and Telecommunications Engineering \\ University of Wollongong, Wollongong NSW 2522 Australia \\ E-mail:mr436@uow.edu.au \\ CSIRO ICT Centre, Marsfield NSW 2122 Australia \\ E-mail: Mark.Hedley@csiro.au
}

\begin{abstract}
Co-operative localisation in wireless sensor networks (WSNs) is a method in which wireless sensor nodes interact with each other as peers to determine their physical position. In such networks it is crucial for communication between nodes to be contention free to meet the strict timing requirements. Medium Access Control (MAC) protocols provide the strongest influence over contention control in WSNs. The focus of this work is the design and analysis of a WSN MAC protocol suitable for supporting co-operative localisation. This paper presents a design overview of the MAC protoco and details its control structure. Preliminary simulation results are also presented to evaluate network formation aspects of the protocol.
\end{abstract}

\section{INTRODUCTION}

Recent advances in wireless communications and microelectronic systems have motivated the development of extremely small, low-cost sensors with sensing, signal processing and wireless communication capabilities. The use of WSNs spans many fields such as military, fire fighting, environment monitoring and home security. In many of these applications sensor nodes are deployed in an ad-hoc manner. In such applications, automatic localisation of the sensors is a key enabling technology. Co-operative localisation can enable many useful applications, such as animal tracking and control and monitoring of manufacturing, production and supply chains [1]. In general, in a co-operative localised network, nodes exchange ranging probes [2]. These probes have to be exchanged in a contention free manner and in periodic tightly timed clusters. A delayed probe or an errored probe will give inaccurate position information.

Co-operative localisation places rigid requirements on access and contention control employed by a MAC protocol. The fundamental task of a MAC protocol is to regulate access to a shared medium to satisfy certain application-dependent performance requirements. In WSNs limited energy resources provide the primary constraint for sensor network protocol design. MAC protocols proposed in the literature primarily focus on reducing energy consumption due to the shared medium contention. Other design constraints, such as fairness, latency, and throughput, appear for specific applications.
The rest of the paper is organised as follows. In Section II we present a short overview of common WSN MAC protocols, focusing on hybrid CSMA/TDMA protocols. In Section III we present the main features of the WiMedia MAC protocol that forms the foundation for our development of a WSN MAC supporting localised co-operation. Section IV discusses localised co-operation requirements and our MAC design approach. Section V presents preliminary results for the performance of the network formation algorithm in the proposed MAC protocol.

\section{WSN MAC PROTOCOLS}

The most widely used class of MAC protocols are contention based. A classical example is the carrier sense multiple access (CSMA) protocol [3]. In CSMA, a node listens to the channel before transmitting. If it detects a busy channel, it delays access and retries at a later time. The simplicity of this approach is that it requires no central controller and there is no need for clock synchronization. Devices joining or leaving a network are handled without many operations and with little complexity. These advantages however, come at the cost of access collisions between two or more nodes trying to transmit at the same time. Moreover, collisions can occur between any two-hop neighbours resulting in the well known hidden terminal problem. Although there are mechanisms to overcome these shortcomings (e.g. RTS/CTS handshake used in IEEE 802.11), they introduce significant overheads [4].

Another class of MAC protocols is based on schedules. These include TDMA, FDMA and CDMA based MAC protocols [5]. They avoid interference by scheduling nodes onto different sub-channels that are divided either by time, frequency or orthogonal codes. Since these sub-channels do not interfere with each other, MAC protocols in this group are largely collision-free. TDMA based protocols have also been adopted in WSNs. TDMA handles the hidden terminal problems well without extra overhead as different nodes can transmit in a contention free manner [6]. However, scheduling of nodes in a scalable manner is not easy. A central node is commonly used to work out a collision free schedule for its neighbourhood. Furthermore, developing a schedule with a 
high degree of concurrency or channel reuse is very difficult [7]. TDMA based protocols also require tight synchronization, which may incur high energy overhead because of frequent message exchanges [4]. These overheads are particularly high in frequent topology changes.

The disadvantages in both scheduled and unscheduled MAC protocols have led to the development of hybrid MAC protocols. SMAC [8] is a distributed hybrid protocol built upon the IEEE 802.11 MAC with an added TDMA structure to incorporate energy saving mechanisms. TDMA is used for scheduled hibernation and contention is used for medium access. To reduce energy consumption in listening to an idle channel, nodes periodically sleep using a TDMA-like scheme. Neighbouring nodes form virtual clusters that autosynchronize on sleep schedules. RTS/CTS handshakes and a back-off mechanism is implemented to contend for the medium in a collision free manner for transmission and reception. Nodes not involved in a transaction set their radios to sleep. SMAC applies message passing to reduce contention latency for sensor-network applications that require store-andforward processing as data move through the network. Advantages of SMAC include low energy waste caused by idle listening which is reduced by sleep schedules, implementation simplicity and avoidance of time synchronization overhead by using sleep schedule announcements. Its disadvantages include lack of RTS/CTS for broadcast data and use of adaptive listening if the packet is not destined to the listening node. Use of predefined and constant sleep and listen periods has been rectified in the revised version of the protocol. The TDMA schedules are now controlled by a higher layer giving the protocol adaptive duty-cycle control, based on contention in the network [9].

Zebra-MAC (Z-MAC) [4] is another recently proposed hybrid TDMA/CSMA protocol. It assigns sensor nodes a time slot but also allows sensor nodes to utilise slots that they do not own through CSMA with prioritised back-off times. This provides Z-MAC with the capabilities similar to CSMA when applications generate less traffic but approximates a strict TDMA scheme when traffic requirements increase. A distributed slot assignment protocol (DRAND) based on the RAND algorithm [10] provides sensor nodes with the time slots they may utilise for transmission and reception. The schedule ensures that two-hop neighbours do not get assigned the same slot number. During each time slot a sensor node uses CSMA to determine if it may transmit when the channel is free (even when it is not its slot to transmit), hence utilising channel bandwidth. However, Z-MAC gives the slot owner preference in channel access by increasing the initial back-off time for sensor nodes that do not own the slot [4]. The advantages of Z-MAC are easy and rapid adaptability to traffic conditions leading to significant energy saving. Compared to other protocols, Z-MAC requires fewer processing and memory resources. Developing a TDMA schedule using DRAND, however, consumes time and energy during network setup. A costly schedule recalculation procedure must be performed for any significant change in the network.
The IEEE 802.15.4 MAC [11] was created for small devices that consume low power and require lower data rates. It supports a centralised star topology and a distributed peerto-peer topology. Regardless of the topology deployed in the network, a Personal Area Network (PAN) co-ordinator is required to control device association within the network. In the peer-to-peer mode, devices will operate independently without a PAN co-ordinator but all devices have to associate with a PAN co-ordinator prior to participating in the network. Devices may operate in a beacon-enabled slotted channel access mode, where the PAN co-ordinator periodically broadcasts a beacon for synchronisation and management purposes, or in an unsynchronised unslotted channel access mode without beacons. In slotted access, devices wait until there is no activity on the channel for two consecutive slots after the initial back-off period, then transmit their messages. Any time a device detects channel activity during the contention procedure, it performs the back-off algorithm and begins the process again at a later time. In the slotted access beacons and an optional superframe structure is used. Beacons are transmitted in the beginning of the superframe. The reminder of the superframe can then be used for contention based as well as contention free access if the optional Guaranteed Time Slots are used The standard provides a lot of detail for the star-topology but does not define clearly the peer-to peer topology. Other problems include the use of beacon-enabled multi-hop networks. There is no proper scheme in the protocol to support multi PAN coordinator synchronisation. This synchronization is necessary as beacon collision will result in loss of data in the control portion of the superframe. Other problems associated with the protocol include the hidden-node problem in largescale networks, the use of Guaranteed Time Slots in time sensitive sensor networks and the suitability of the Zigbee routing layer with the IEEE 802.15.4 MAC.

\section{WIMEDIA MAC}

The WiMedia Alliance has developed a MAC protocol for ultra wideband based wireless networks, although not specifically targeted for WSNs. The WiMedia MAC protocol is a fully distributed TDMA/CSMA protocol also designed to handle high mobility scenarios [12]. The MAC enables a distributed clock synchronisation via self-beaconing devices, thus minimising uncoordinated transmissions. More importantly, the distributed nature of the protocol provides full mobility and QoS supports. All devices perform identical functionality using local information only.

The WiMedia MAC protocol uses TDMA for periodic intervals for operations between devices. The superframe is divided into 256 Medium Access Slots (MAS). A superframe is composed of two parts: a beacon period (BP) and a data transfer period (DTP). The superframe starts with a BP which extends over one or more contiguous MAS. During the BP, each device sends its beacon at its time slot with network information. Other devices are always listening during the BP. Therefore even in idle mode, the receiver is on for the entire duration of the BP. However for energy efficiency, the BP 
length is dynamically adapted to the shortest possible duration supporting the maximum allowable number of devices. During a typical BP, a device first wakes up and starts to scan the channel to detect existing BPs. If a BP is detected, the device will try to synchronise to it, otherwise it will create its own BP. The BP is divided into slots. The first two slots are used for signalling purposes. A new device wanting to join the network has to acquire a unique slot in the BP. If the choice of the slot extends over the BP length, the new device has to send a beacon in the signalling slot informing the need for extending the BP length.

Following the BP is the Data Transfer Period where devices use Distributed Reservation Protocol (DRP) and/or Prioritised Channel Access (PCA) for data transmission. The PCA mechanism provides the contention access to the medium and improves the standard CSMA introducing the access categories and transmission opportunities. The DRP is a distributed protocol to reserve MASs for isochronous traffic. The WiMedia superframe structure is shown in Figure 1.

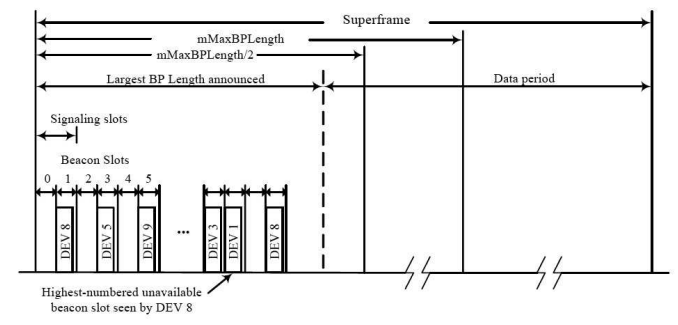

Figure 1: WiMedia Superframe Structure. [12]

To maintain time synchronization, each device maintains a beacon period start time (BPST). All devices adjust their BPST to maintain superframe synchronisation with its slowest neighbour. Only after synchronisation is achieved will the protocol allow for the device to transmit its first beacon. Upon receiving a beacon from a neighbour, a device will determine the difference between the beacon's actual reception time and the expected reception time. The actual received time is estimated to be the time of arrival of the beacon's preamble. The expected reception time is determined from the beacon slot number field of the received beacon and the receiving beacon's BSPT. To maintain the superframe synchronisation with the slower neighbour, the receiving device will delay its BPST by the difference calculated but this delay is limited to a specific length, predetermined prior to network activation, per superframe. All adjustments made to BPST have to be done before the end of a superframe.

Although the WiMedia MAC does not specifically target WSNs, it contains features that can be adapted to create a simplified MAC protocol suitable for supporting co-operative localisation in distributed network topologies.

\section{MAC PROTOCOL DESIGN}

\section{A. Co-operative Localisation Requirements}

Our research in co-operative localisation is targeted to applications such as emergency services. Characteristics of such applications are that there will only be a small number of anchor nodes at which location is known, and a large number of nodes forming the network with many of them being attached to people or vehicles that may be highly mobile. The algorithm to perform the co-operative localisation has two stages. In the first an initial estimate of the node locations is formed. The second stage is an iterative optimisation of the non-linear equations to refine the computed locations. Details on these algorithms are given in [13].

Co-operative localisation imposes two requirements on the MAC, the transmission of the ranging probes and data exchanges for the computation of the node locations. The requirements on the MAC for the first are:

- Rapid adaptation to frequent topology changes due to highly mobile nodes.

- Transmissions of periodic ranging probes are to be scheduled in such a way that they are received as tightly time clustered probes by each node.

- Probes should be sent in reserved time slots (probes are broadcast, not targeted to a particular node). Acknowledgements to received probes are not provided as it is undesirable to have retransmissions of probes.

The data exchange requirements for the computation of location depend upon where the location is computed and the tolerable latency in the availability of the data. In the current implementation each node sends the information from received probes to a central location server that performs a global optimisation. To provide real time location information the MAC (and higher layers) must minimise the time taken to route through the ad hoc network to the location server. Centralised computation can provide better results than distributed computation, however while it can handle networks containing tens to perhaps hundreds of nodes it does not scale to larger networks. Our current research [13] is focusing on developing algorithms for distributed computation with good performance and low computation requirements. As multiple packets will need to be exchanged within neighbourhoods to run iterative algorithms low latency will be an essential requirement on the communication protocol.

\section{B. MAC Design Approach}

The need for periodic transmissions in a contention free manner points to a need for a TDMA system. However, TDMA networks do not perform very well without a coordinator. Developing an efficient schedule with a high degree of concurrency or channel reuse is difficult. Moreover factors such as interference, time varying channel conditions and clock synchronisation errors diminish the benefits of 
TDMA. The need for a distributed network topology and the ability to cope with non-periodic transmissions suggests the need for a CSMA system. However, CSMA systems suffer from large overheads as the size of the network increases. Based on the above considerations, we require a hybrid MAC protocol which provides both TDMA and CSMA functionality. We have selected the WiMedia MAC as the foundation for developing a MAC suitable for co-operative localisation. Although the WiMedia MAC is not a WSN $\mathrm{MAC}$, its functionality can be adapted to the requirements of co-operative localisation.

\section{MAC Protocol Design}

The adopted design aspects from the WiMedia MAC are as follows:

- General Superframe structure consisting of Beacon Period and Data Transfer Period

- Beacon exchange mechanism

- Beacon period contraction

- Collision detection

- Time synchronization

To meet the requirements of co-operative localisation, changes were made to the following WiMedia MAC protocol features:

- Beacon period structure

$\circ \quad$ No signalling slots

- Same time slot size for both Beacon Period and Data Transfer Period

- Beacon structure and information content

○ Varying length beacon frames for different scenarios

- Contention resolution

- Random back-off mechanism

- Introduction of new rules for:

- Packet loss

- Asymmetric link resolution

- Link formation

\section{Beacon Period}

There is no central coordinator in the network and the BP functions as the point of entry for all nodes in the network. It limits the maximum density of nodes in the network. Nodes exchange information about their two-hop neighbourhood and request other services (e.g. a slot number change) in the beacon slot. Nodes in the two-hop network contend to gain access to the channel. This process of slotted exchange of information takes place at the start of the superframe in the BP. Nodes in the BP exchange beacon information. Figure 2 shows a typical beacon frame. The beacon Header contains the length of the entire beacon frame and the length of the Slot Occupied Information. Device ID is the node's device address. Slot Number is the node's current beacon slot number. The content of the Slots Occupied Information frame depends on the status of the node. Figure 2 shows the content of the Slot Occupied Information frame for a node after the network has formed and all nodes have succeeded in obtaining their beacon slots (i.e. contention has been resolved). The frame in this case consists of the slot numbers, status information and device IDs of its neighbours. Table 1 summarises possible beacon's Slot Status states.

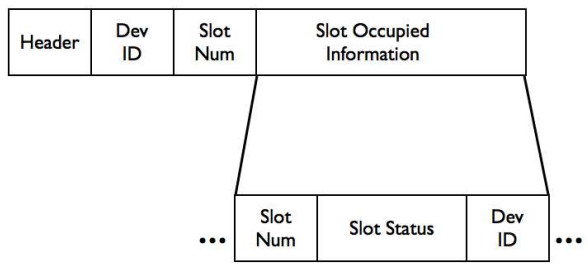

Figure 2: Typical Beacon Frame

\begin{tabular}{|c|l|}
\hline $\begin{array}{c}\text { Element } \\
\text { ID }\end{array}$ & \multicolumn{1}{|c|}{ Beacon Slot Status } \\
\hline 0 & $\begin{array}{l}\text { Occupied (non movable). Set by nodes when they are active } \\
\text { in their slot and wish not to move to an earlier slot. }\end{array}$ \\
\hline 1 & $\begin{array}{l}\text { Occupied Moving. Sent out by one-hop neighbours to } \\
\text { indicate a beacon slot movement by the node associated with } \\
\text { the slot status. }\end{array}$ \\
\hline 2 & $\begin{array}{l}\text { Low PHY Activity. A PHY indication of medium activity } \\
\text { was received in the corresponding beacon slot in the last } \\
\text { superframe but did not result in reception of a frame with } \\
\text { valid beacon information in this superframe. }\end{array}$ \\
\hline 3 & $\begin{array}{l}\text { Requesting Slot. Sent by new nodes wishing to join the } \\
\text { network. }\end{array}$ \\
\hline 4 & $\begin{array}{l}\text { Tentative. A beacon slot is set to tentative by a neighbour(s) } \\
\text { accepting re-quest(s) for a slot use. }\end{array}$ \\
\hline 5 & $\begin{array}{l}\text { Contention. A beacon slot is set to Contention if more than } \\
\text { one node contends for it. This flag is set by neighbours that } \\
\text { listened to the request for slot placement. }\end{array}$ \\
\hline
\end{tabular}

Table 1: Beacon Slot Status.

\section{E. Network Formation Algorithm}

Network formation involves a node listening to the network to learn about which slots are empty, selecting the earliest time slot and then resolving possible contention for that time slot. This algorithm is shown in Figure 3 and Figure 4. The main parameters of interest in the algorithm are the maximum number of available beacon slots and the maximum back-off number (in number of superframes) used in the back-ff procedure. Figure 3 and Figure 4 show the steps involved in the algorithm. 


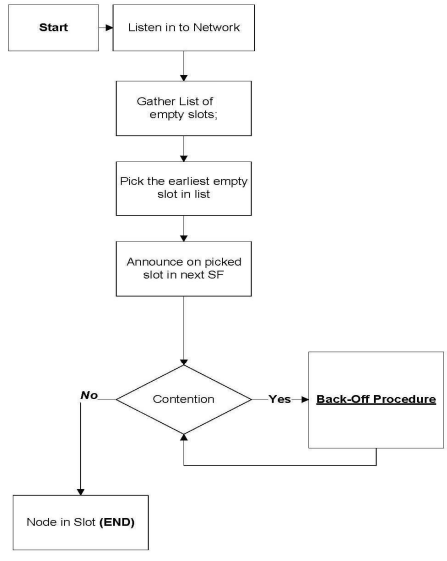

Figure 3: Network Formation Algorithm Main Loop

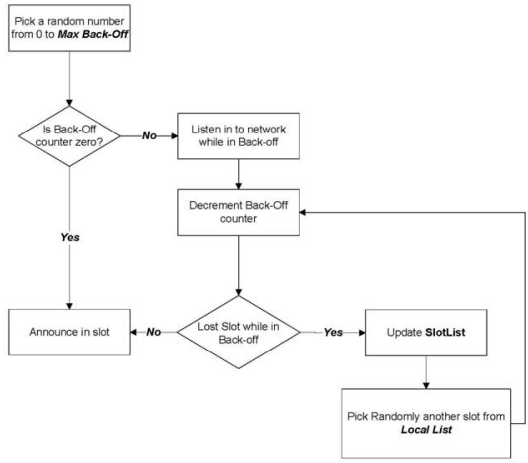

Figure 4: Network Formation Algorithm Back-Off Procedure

\section{SIMULATION RESULTS}

We have simulated the network formation algorithm to determine the amount of time it takes for the MAC protocol to resolve the contention during the network start-up and when new nodes join the network. The network formation algorithm was simulated in $\mathrm{C}++$.

Figure 5 shows the number of superframes it takes for the network to form as a function of the maximum number of back-offs allowed for different combinations of the number of nodes and the number of slots in the beacon period. It can be seen that the maximum number of back-offs of between two and three superframes leads to the fastest network formation in these cases. In addition, as can be seen form the figure, this choice of the maximum number of back-offs was found to be quite insensitive to the number of nodes and the number of beacon slots used. The algorithm appears to perform best with the choice of two for the maximum back-off value for small networks and with the choice of three for the maximum backoff value for large networks.

Figure 6 shows the number of superframes it takes for the network to form as a function of the number of beacon slots in a network of 500 nodes. Figure 7 shows the corresponding results as a function of the number of nodes in a network using 500 beacon slots. In both cases, the maximum number of back-offs used was set to three. Each experiment was repeated 100 times with a random seed and the results are displayed with $95 \%$ confidence intervals.

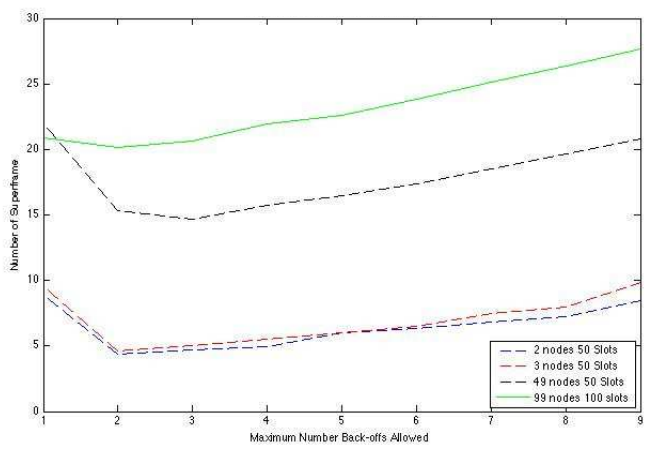

Figure 5: Number of Superframes Required for Network Formation vs Maximum Number of Back-Offs Allowed for Various Network Sizes and Numbers of beacon Slots.

Figures 6 and 7 provide a trend perspective for the network. The figures show an almost linear relation between the number of superframes it takes for the network to become contention free and the number of nodes/beacon slots. The limiting factor for the network is the number of beacon slots. This limits the number of nodes that can join a two-hop neighbourhood. Both the random back-off and the random slot allocation values chosen by a node belong to a uniform distribution. Further analysis with values chosen from different distributions might provide insights to improve system performance. However, results obtained thus far can be used as benchmarks for future optimisation. Also, if the localisation algorithm requires a network to become contention free within a certain number of superframes, these results can provide the constraints on the maximum number of nodes and beacon slots in the network. 


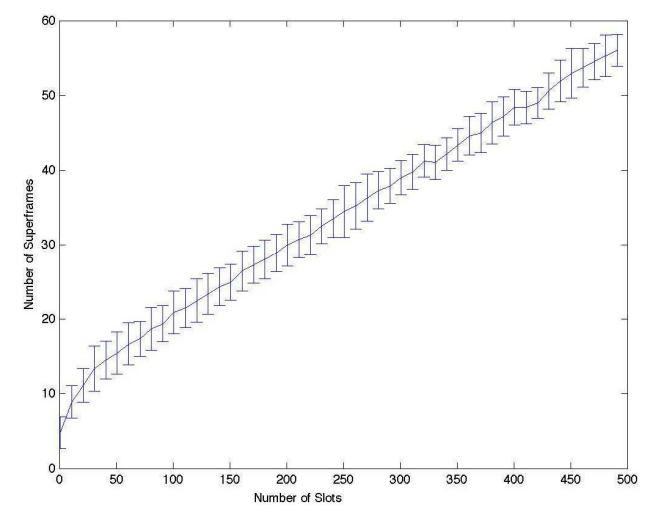

Figure 6: Number of Superframes Required for Network Formation vs Number of Beacon Slots for a 500 Nodes Network

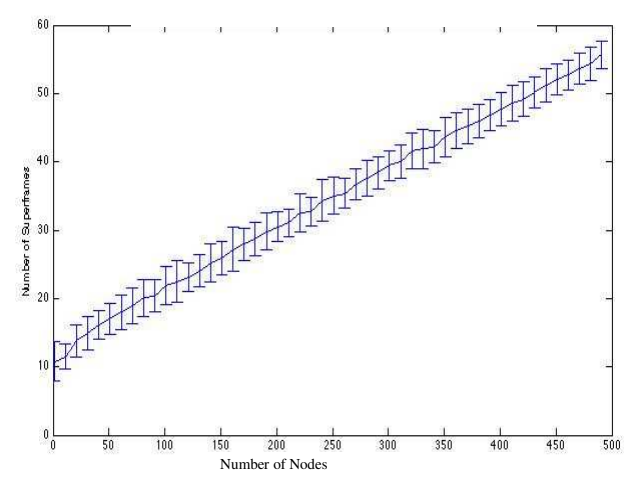

Figure 7: Number of Superframes Required for Network Formation vs Number of Nodes in the Network using 500 Beacon Slots

\section{CONCLUSIONS}

In this paper we discussed the requirements of co-operative localisation on WSN MAC design. A review of popular MAC protocol was made to highlight their salient features and suitability for supporting co-operative localisation. The WiMedia MAC was selected as a suitable starting point for development of a MAC protocol to support co-operative localisation in distributed network topologies. We outlined how the WiMedia MAC can be adapted and we provided the main features of the resulting WSN MAC protocol. In this paper we also presented the details of the network formation algorithm in the resulting WSN MAC protocol. Simulation results showed the impact of the maximum back-off number, the network size and the number of beacon slots on the network formation time. Future work will focus on the development of efficient beacon contraction and cluster merger algorithms.

\section{REFERENCES}

[1] N. Patwari, J.N. Ash, S. Kyperountas, A. O. Hero III, R. L. Moses and N. S. Correal, "Locating the Nodes: Co-operative localization in wireless networks," IEEE Signal Processing Magazine, pp. 54-69, July 2005.

[2] L. Hu and D. Evans, "Localization for mobile sensor networks," Tenth Annual International Conference on Mobile Computing and Networking, October 2004.

[3] A. W. Holger Karl, Protocols and Architectures for Wireless Sensor Networks. Wiley, 2005.

[4] A. Warrier, I. Rhee, M. Aia and J. Min, "Z-MAC: a hybrid MAC for wireless sensor networks," SIGCOMM, 2005.

[5] J. Schiller, Mobile Communication. Addison Wesley, 2003.

[6] K. Sohrabi and G.J. Pottie, "Performance of a novel selforganization protocol for wireless ad hoc sensor networks," IEEE $50^{\text {th }}$ Vehicular Technology, pp. 1222-1226, 1999.

[7] K. Sohrabi, J. Gao, V. Ailawadhi and G.J. Pottie, "Protocols for self-organization of a wireless sensor network," IEEE Personal Communications, pp. 16-27, October 2000.

[8] W. Ye and J. Heidemann, "An energy-efficient MAC protocol for wireless sensor networks," IEEE Infocom, pp. 1567-1576, June 2002.

[9] Wei Wang and Dongming Peng and Honggang Wang and Hamid Sharif, "Study of an energy efficient multi rate scheme for wireless sensor network MAC protocol," Proceedings of the 2nd ACM International Workshop on Quality of Service \& Security for Wireless and Mobile Networks, pp. 51-54, 2006.

[10] I. Rhee, A. Warrier, J.K. Min and L. Xu, "DRAND: distributed randomized TDMA scheduling for wireless ad-hoc networks," Proceedings of the seventh International Symposium on Mobile Ad Hoc Networking \& Computing, pg. 190-201, 2006.

[11] LAN-MAN Standards Committee of the IEEE Computer Society, "Wireless Medium Access Control (MAC) and Physical Layer (PHY) Specifications for Low-Rate Wireless Personal Area Networks (WPANs)," IEEE, 2006.

[12] Standard ECMA-368, High Rate Ultra Wideband PHY and MAC Standard, http://www.ecma-international.org/, December 2005.

[13] F. Engel and M. Hedley, "A comparison of cooperative localization techniques for wireless mobile sensor networks," ISCIT 2007. 